\title{
Tagungsbericht
}

Saskia Dorschel, Ulrike Ostrzinski* und Elke Roesner

\section{Allen Widrigkeiten zum Trotz: „Zukunft Wissenschaftlicher Bibliotheken?!“}

\author{
Bericht und Blick hinter die Kulissen der virtuellen Fachtagung von ZB MED und Open \\ Password am 18. Mai 2020
}

https://doi.org/10.1515/iwp-2020-2106

Tagungen im virtuellen Raum finden derzeit permanent statt und die Lernkurve ist recht hoch. Daher verfolgen wir mit diesem Tagungsbericht zwei Ziele: Zum einen geben wir einen kurzen Überblick über die Inhalte der Veranstaltung, die zukünftige Herausforderungen Wissenschaftlicher Bibliotheken in den Fokus nahm. Zum anderen berichten wir über die Organisation im Hintergrund. Wir möchten Sie gerne an unseren Erfahrungen und Erkenntnissen teilhaben lassen. Dazu haben wir ein paar nützliche Tipps für virtuelle Tagungen zusammengestellt.

Aus ZB MED-Sicht berichten wir über eine Erfolgsgeschichte - im Hintergrund haben wir alle möglichen Höhen und Tiefen durchlaufen und am Ende können wir zufrieden auf eine rundum erfolgreiche Fachtagung am 18. Mai 2020 zurückblicken.

\section{Es begann ganz normal und dann...}

Bereits im November verschickten ZB MED - Informationszentrum Lebenswissenschaften und das Fachmagazin Open Password die Einladung zu ihrer gemeinsamen Veranstaltung zur Zukunft Wissenschaftlicher Bibliotheken am 23. Januar 2020 am Kölner Standort von ZB MED. Der Zuspruch war rege und wir bereiteten uns auf eine gut besuchte Tagung vor. Am späten Nachmittag des 22. Januar kam dann die Meldung: Bombenfund auf dem Gelände der Uniklinik in Köln! Erst in den frühen Abendstunden

\footnotetext{
Saskia Dorschel, ZB MED - Informationszentrum Lebenswissenschaften, Gleueler Straße 60, 50931 Köln,

E-Mail: marketing@zbmed.de

*Kontaktperson: Ulrike Ostrzinski, ZB MED - Informationszentrum Lebenswissenschaften, Gleueler Straße 60, 50931 Köln, E-Mail: marketing@zbmed.de

Elke Roesner, ZB MED - Informationszentrum Lebenswissenschaften, Gleueler Straße 60, 50931 Köln,

E-Mail: marketing@zbmed.de
}

war klar, dass die Entschärfung am nächsten Tag erfolgen sollte und von der großflächigen Räumung des Areals auch das Gebäude von ZB MED betroffen wäre. Es blieb keine Wahl - die Veranstaltung musste abgesagt werden. In einer abendlichen Aktion haben wir die bundesweit anreisenden Vortragenden und die große Zahl der Teilnehmerinnen und Teilnehmer sowie die Dienstleister über verschiedene Kanäle informiert und zum Glück die allermeisten auch erreicht. Das Verständnis für die erzwungene Absage war da. Gleichzeitig wurde ein allgemeines Bedauern über die abgesagte Tagung geäußert. Auch wir vom Organisationsteam waren sehr enttäuscht, dass unsere dezidiert geplante Tagung so nicht stattfinden konnte.

Schnell stand der 18. Mai 2020 als neuer Termin fest und wir planten alles um, verschickten erneut die Einladungen, nahmen Anmeldungen entgegen.

Und dann erreichte die COVID-19-Pandemie auch Deutschland; das warf alle Planungen und Vorerfahrungen über den Haufen. Nun konnten nicht einmal Treffen im kleinen Kreis, geschweige denn größere Veranstaltungen stattfinden. In einem waren sich alle schnell einig: Die Fachtagung sollte nicht ausfallen! Kurzerhand beschlossen wir gemeinsam mit Willi Bredemeier von Open Password die Tagung in einen virtuellen Konferenzraum zu verlegen. Wir hatten bei ZB MED schon in der ersten Woche des Lockdowns Zoom-Meetings mit fast der gesamten Belegschaft durchgeführt. Wir wussten, dass hier viel möglich ist. Die Digitalisierung ist nicht ohne Grund ein wichtiger Baustein in den Strategien der Bibliotheken. Und diese besondere Zeit der COVID-19-Pandemie bietet eine Chance, große Fortschritte in der Umsetzung zu machen. Das Thema der Konferenz erwies sich als noch aktueller als ursprünglich gedacht. Denn die besondere Situation zeigt noch deutlicher als ohnehin bekannt, wie sehr die Thematik der Zukunftsfähigkeit Wissenschaftlicher Bibliotheken unter den Nägeln brennt. 


\section{Die Themen der Tagung - aktueller als gedacht}

In der digitalen Transformation stehen Bibliotheken ebenso wie ihre Klientel an den Hochschulen und Forschungseinrichtungen vor großen Herausforderungen: Open Access, Forschungsdatenmanagement, Informations- und Datenkompetenz, Langzeitarchivierung, Künstliche Intelligenz, Citizen Science, Personalgewinnung als neuer Engpass, die Anpassung der bibliothekswissenschaftlichen Lehre an neue Anforderungen - um nur diese zu nennen. Einige Wissenschaftliche Bibliotheken wie zum Beispiel ZB MED oder TIB begegnen dem mit der Intensivierung ihrer Forschung und stehen dadurch in einem bedeutenden Strukturwandel. Die Fachtagung schließt an das 2019 erschienene Buch „Zukunft der Informationswissenschaft Hat die Informationswissenschaft eine Zukunft?“ an. Da das Buch als zentrales Thema auch die Perspektiven Wissenschaftlicher Bibliotheken beleuchtet, initiierte der Herausgeber Dr. Willi Bredemeier die Fachtagung mit ZB MED, um die Diskussion fortzusetzen.

\section{Wie bringt man eine analog geplante Tagung in die virtuelle Welt?}

Um aus einer Präsenztagung eine virtuelle Tagung zu machen, mussten wir einige Dinge beachten. Zunächst stellte sich die Frage der Technik. Mit welchem Programm können wir den Tag durchführen? Welche Voraussetzungen müssen wir im Team dafür schaffen und welche Voraussetzungen können wir den Teilnehmenden zumuten? Wir entschieden uns für die Plattform Zoom, da wir selbst bei größeren Veranstaltungen damit bessere Erfahrungen als mit anderen Konferenztools sammeln konnten. Gespräche mit Kooperationspartnerinnen und -partnern bestätigten unsere Einschätzung. Hinzu kam unserer Vermutung, dass Zoom durch die hohe Verbreitung auch im universitären Umfeld bei den meisten Teilnehmenden bereits genutzt wird. Klar war uns auch, dass es sicherlich einige geben würde, die wegen des Tools nicht teilnehmen könnten - aber eine Plattform zu finden, die für alle passt, ist kaum möglich.

Mit der wachsenden Zahl der Anmeldungen wurde schnell deutlich, dass wir unseren vorhandenen Zoom-Account upgraden mussten. Wir stockten die zugelassene Teilnehmendenzahl kurzfristig auf. Bei einigen internen Tests im Organisations-Team machten wir uns vertraut mit der Technik, den einzelnen Abläufen und den jeweiligen Darstellungen aus Teilnehmenden- und Veranstaltersicht.

\section{Wir legten Regeln für die virtuelle Fachtagung fest}

Alle Mikros und Videos der Teilnehmenden sollten während der kompletten Tagung ausgeschaltet sein. Zudem konnten sie ihre Bildschirme nicht teilen. Ziel war es, ungewollte Geräusche/Bilder zu vermeiden und die Übertragung der Fachtagung stabil zu halten. Die Kommunikation - Fragen und Anmerkungen der Teilnehmerinnen und Teilnehmer - verlegten wir in den Chat als Ersatz für die sonst übliche Diskussion mit dem Publikum.

Auf der anderen Seite sollten alle Referentinnen und Referenten sowie das Organisations-Team ihr Video einschalten, damit die Atmosphäre eines Auditoriums geschaffen wird und niemand ausschließlich in einen schwarzen Bildschirm sprechen muss.

Für die Gewährleistung eines möglichst reibungslosen Verlaufs und um auf unplanbare Eventualitäten reagieren zu können, bekamen das gesamte OrganisationsTeam sowie alle Referentinnen und Referenten die erweiterten Rechte als Co-Host zugewiesen. Das ermöglicht u.a. das Eingreifen bei Problemen, die Bildschirmfreigabe, um eine Präsentation abzuspielen, das gegenseitige Stummoder Lautschalten etc.

Einige Tage vor der Konferenz durchliefen wir in einer Art Generalprobe mit fast allen Referentinnen und Referenten kurz einige Eckpfeiler der Tagung. Es ging primär darum, den Ablauf abzustimmen, die Technik von Zoom $\mathrm{zu}$ testen und individuelle Fragen zu beantworten. Vorab hatten wir einen präzisen Ablaufplan aufgestellt, welcher nicht nur die zeitliche Reihenfolge der Vorträge auflistete, sondern auch bestimmte, wann wer welche technischen Abläufe ausführen sollte. Es war also zum Beispiel genau festgelegt, wann der virtuelle Konferenzraum geöffnet wird, wann die Begrüßungsfolie eingeblendet wird, wer einen Filmbeitrag startet und wann wer wen im Spotlight-Modus freischaltet.

Innerhalb des sechsköpfigen Organisations-Teams gründeten wir als zweiten Kommunikationskanal eine Messenger-Gruppe, um am Durchführungstag bei Problemen schnell reagieren oder um Hilfe bitten zu können. Um auf technische Probleme der Teilnehmenden während der Tagung eingehen zu können, verschickten wir vorab eine E-Mail-Adresse, an die man sich wenden konnte. Das entsprechende Postfach hielten während der Veranstaltung 


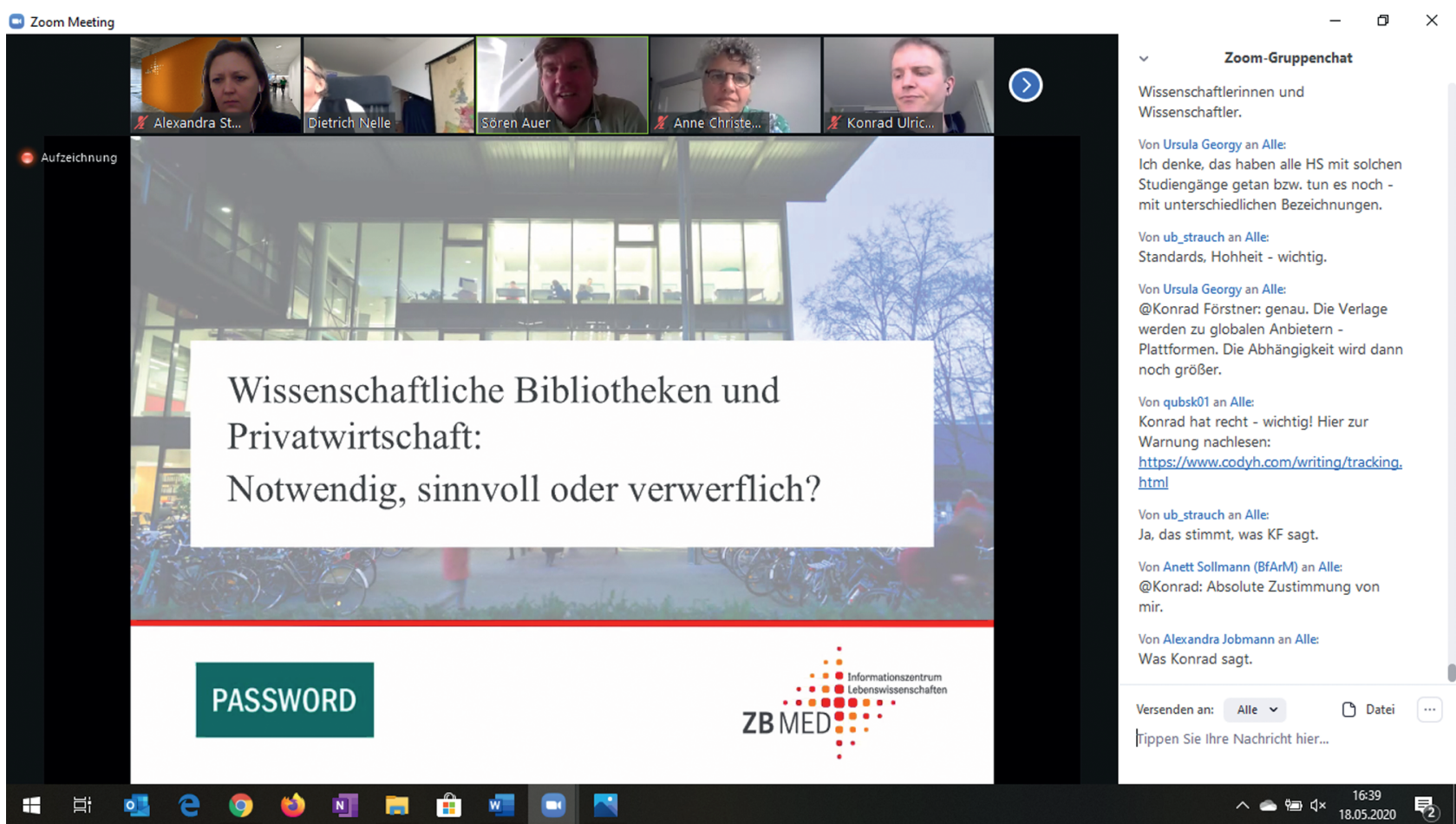

Abbildung 1: Screenshot mit Alexandra Streck aus dem Organisationsteam sowie den Referentinnen und Referenten Dr. Dietrich Nelle, Prof. Dr. Sören Auer, Anne Christenden, Prof. Dr. Konrad Förstner (von links nach rechts).

immer zwei Personen aus dem Organisations-Team im Blick. Eine weitere Person aus dem Team war dafür zuständig, per Mail- und Telefonsupport den Referentinnen oder Referenten bei Problemen weiterzuhelfen.

\section{Umgestaltung eines analogen Programms in die virtuelle Welt}

Bei der Planung wurde uns schnell klar, dass wir das Programm etwas umgestalten mussten. Zunächst brauchten wir eine Person, die die virtuelle Fachtagung moderiert und sich gleichzeitig in der Technik sicher fühlt. Dafür konnten wir unsere Kollegin Dr. Miriam Albers gewinnen, die zugleich als Dozentin an der TH Köln tätig ist und so hinreichende Vorerfahrungen mit (virtuellen) Vorlesungen hat. Um mehr Raum für die Beantwortung von Fragen aus dem Chat zu haben, kürzten wir die Zeit der geplanten Podiumsdiskussion und gaben den Referentinnen und Referenten fünf Minuten zusätzlich.

Unsere These: Eine Diskussionsrunde über Zoom zu führen ist äußerst schwierig. Somit ersetzten wir die ohnehin schon gekürzte abschließende Podiums-Diskussion durch vier Statements, zu denen die Referentinnen und Referenten abwechselnd Stellung nehmen sollten.

\section{Planung des zeitlichen Ablaufs}

Damit die Fachtagung im zeitlichen Rahmen blieb, brauchten wir eine Person für die Zeitüberwachung. Unsere Moderatorin Miriam Albers erklärte sich auch zu dieser Aufgabe bereit und hielt immer vor Ablauf der letzten 5 Minuten einen Zettel in die Kamera. Für diese Aufgabe hatten wir zudem ein Backup eingeplant. Wenn die Referentinnen oder Referenten doch überzogen hätten, wäre nach Ablauf ihrer Zeit ein Handy-Klingelton zu hören gewesen, da es nicht immer gelingt, über optische Signale Menschen im Redefluss über Zoom zu unterbrechen. Glücklicherweise kam dieses Mittel nicht zum Einsatz. Selten sind wir bei einer Veranstaltung so genau im Zeitplan geblieben - vermutlich dank der besonders genauen zeitlichen Planung und den guten Vorab-Instruktionen!

\section{Zukunft Wissenschaftlicher Bibliotheken?! - Kernaussagen der Tagung}

In einer Keynote und mehreren Impulsvorträgen gaben Expertinnen und Experten aus unterschiedlichen Blickwinkeln Anregungen und stellten zukunftsweisende Projekte und Entwicklungen vor. 
Den Anfang machte Dr. Dietrich Nelle vom BMBF, ehemaliger Interimsdirektor von ZB MED und VDB-Ehrenmitglied. Besonderes Augenmerk legte er auf den Bereich Open Access und die DEAL-Verhandlungen, bei denen bereits Durchbrüche erzielt werden konnten. Auch das BMBF ist im Bereich Open Access aktiv, beispielsweise mit einer Kompetenz- und Vernetzungsstelle, die in Zukunft die Open-Access-Community effektiv unterstützen wird. Mit der Zunahme von Open Access und in der fortschreitenden Digitalisierung ändert sich das Aufgabenspektrum von Bibliotheken. So entsteht zum Beispiel der Bedarf für eine Publikationsinfrastruktur, neue Publikationsformate, Forschungsdatenmanagement und digitale Langzeitarchivierung. Bibliotheken selbst müssen eine aktive, gestalterische Rolle einnehmen. Hier unterstrich Dr. Nelle die Bedeutung von Kooperationen mit Rechenzentren und auch Digitalfirmen sowie die Zusammenarbeit in nationalen und internationalen Netzwerken.

Anne Christensen, ehemalige Bibliotheksdirektorin, jetzt Beraterin und Mitinhaberin bei effective WEBWORK, stellte fünf Thesen für erfolgreiche Strategien bei der Umsetzung von digitalen Diensten in Bibliotheken auf, die laut der ersten These ins Zentrum der Bibliotheksstrategie gehören. Ihre Forderung: Solche Dienste brauchen WowEffekte! Als Praxisbeispiel zeigte sie die Besetzungssuche der HMT Leipzig als maßgeschneidertes Tool. Anne Christensen betonte wie auch ihr Vorredner die Bedeutung der Zusammenarbeit - von Bibliotheken untereinander, innerhalb der Einrichtungen, mit Dienstleistern, aber auch mit den Nutzenden. Geänderte Bedingungen zeigen sich zudem in einem neuen Informationsverhalten der Zielgruppen und einem anderen Mindset von Mitarbeitenden, die heute häufig auch aus anderen Berufsgruppen kommen. Dies macht eine generelle Offenheit und Bereitschaft zur Diversität erforderlich und stellt das Führungspersonal vor neue Herausforderungen.

Der Arzt und Informatiker Prof. Dr. Dietrich RebholzSchuhmann hat vor zwei Jahren die Wissenschaftliche Leitung von ZB MED übernommen. Sein Ziel: Forschung und Bibliothek arbeiten innerhalb einer Einrichtung auf Augenhöhe zusammen und ergänzen sich dabei gegenseitig. Vergleicht man die Angebote von ZB MED mit den Handlungsfeldern, die das Positionspapier des DBV „Wissenschaftliche Bibliotheken 2025“1 aufführt, zeigt sich, dass ZB MED auf einem guten Weg ist - die dort genann-

1 https://www.bibliotheksverband.de/fileadmin/user_upload/Sekti onen/sektion4/Publikationen/WB2025_Endfassung_endg.pdf [5.6.2020]. ten Dienste laufen entweder bereits oder sind in Planung. Die ZB MED-eigene Forschung im Bereich der Datenwissenschaften spielt bei der Weiter- und Neuentwicklung von Angeboten eine entscheidende Rolle. Im Fokus der Services steht insbesondere die Zielgruppe der Forschenden. So sollen für jedes Stadium im Forschungskreislauf entsprechende Dienste angeboten werden. Besondere Bedeutung hat die Kooperation zwischen ZB MED und dem Institut für Bioinformatik-Infrastruktur in Bielefeld (BIBI). Diese Allianz bietet Lösungen, die das gesamte Spektrum der Informationsversorgung und Datenanalyse abdecken und so neue Forschungserkenntnisse ermöglichen. Am Beispiel des ZB MED COVID-19 Hub² zeigte Dietrich Rebholz-Schuhmann, wie die Zusammenarbeit bei ZB MED in der Praxis aussieht. Der Hub ist Anfang März innerhalb kurzer Zeit in enger Kooperation unterschiedlicher interner Bereiche entstanden. Beteiligt waren Forschende und Promovierende, Bibliothekarinnen und IT-Entwickler sowie das Marketing.

Prof. Dr. Sören Auer, Direktor der TIB - Leibniz-Informationszentrum Technik und Naturwissenschaften aus Hannover, setzte mit seinem Vortrag bei den geänderten Informationsflüssen in der Wissenschaft an. Die bisherigen Prozesse können dem nicht mehr gerecht werden. Als Lösung des Problems zeigte er den Open Research Knowledge Graph, der bei seiner Einrichtung bereits eingesetzt wird. Solche Wissensgraphen reichern Textquellen an, beispielsweise mit bibliographischen Angaben oder Informationen zur Methodik oder der Art der Organismen und verknüpfen sie mit externen Quellen wie zum Beispiel den zugrundeliegenden Forschungsdaten. Die Wissensgraphen liefern bei speziellen Anfragen andere Antworten auf Forschungsfragen und ermöglichen einen schnellen Überblick zu einem Forschungsgebiet. Sören Auer zeigte als Beispiel einen von der TIB entwickelten Open Research Knowledge $\mathrm{Graph}^{3}$ zur COVID-19-Forschung. Denkbar ist der Einsatz jedoch in ganz unterschiedlichen Disziplinen in enger Zusammenarbeit mit den jeweiligen Fachwissenschaftlerinnen und -wissenschaftlern.

Im November 2019 ging der Zertifikatskurs „Data Librarian" als Blended-Learning-Format an den Start. Als wissenschaftlicher und fachlicher Leiter hat Prof. Dr. Konrad Förstner - Professor für Information and Data Literacy an der TH Köln und Leiter der Informationsdienste bei ZB MED - den Kurs gemeinsam mit dem Zentrum für Bibliotheks- und Informationswissenschaftliche Weiterbildung

2 https://www.zbmed.de/covid-19/uebersicht/ [27.5.2020].

3 https://www.orkg.org/orkg/ [27.5.2020]. 
(ZBIW) konzipiert. Zusammen mit Marvin Lanczek vom ZBIW stellte Konrad Förstner den Kurs vor, der sich an Beschäftigte Wissenschaftlicher Bibliotheken richtet, die Chancen und Möglichkeiten der Digitalisierung nutzen und umsetzen wollen. Ziel ist es, Grundkenntnisse in den Bereichen Big Data, Open Science und Forschungsdaten zu vermitteln und so Hemmschwellen abzubauen. Denn es ist unabdingbar, am Ball zu bleiben und permanent Kenntnisse zu vertiefen und neue Skills zu entwickeln. Der Kurs versetzt die Absolventinnen und Absolventen in die Lage, Herausforderungen anzunehmen und qualifiziert sie für neue Aufgaben. Es werden keine Vorkenntnisse benötigt, die Bewerbung erfolgt über ein Motivationsschreiben. Der Zeitaufwand beträgt ca. 210 Stunden einschließlich Präsenztagen und E-Learning-Phasen. ${ }^{4}$

Während der Vorträge nutzten die Teilnehmerinnen und Teilnehmer die Möglichkeit, sich mit Fragen und Anmerkungen im Chat an der Diskussion zu beteiligen. So entstand ein lebhafter Austausch - mit den Vortragenden, aber auch unter den Teilnehmenden.

In der Abschlussrunde, die die geplante Podiumsdiskussion ersetzte, gab es vier teils provokante Thesen, zu denen alle Vortragenden in jeweils zwei Minuten ihre Statements abgaben:

- Wissenschaftliche Bibliotheken zwischen Formalerschließung und Forschungsdatenmanagement: Was ist Kunst und was kann weg?

Alle stimmten überein, dass eine Pauschalantwort auf diese Frage nicht möglich ist. Angebote gehören jedoch auf den Prüfstand: Was fragen die Nutzenden nach und was liefert auch längerfristigen Mehrwert? Es nützt nichts, an Dingen festzuhalten, nur weil man sie immer schon gemacht hat oder der Service gerade en vogue ist. Wichtig ist es, im Blick zu haben, dass hinter tiefgreifenden Veränderungen und Abwicklungen von Angeboten Mitarbeitende mit Trauer und Ängsten stehen, die ernst genommen werden müssen.

- Data Librarian, Systembibliothekar und Co: Welche Qualifikationen brauchen wir und wie lassen sich diese rekrutieren?

Neben Managementkompetenzen und Kommunikationsfähigkeit spielt die Bereitschaft zum lebenslangen Lernen sowie die Selbstlernfähigkeit eine entscheidende Rolle.

4 https://www.th-koeln.de/weiterbildung/zertifikatskurs-data-librar ian_63393.php [5.6.2020].
Dafür ist ein gutes Fortbildungsangebot entscheidend. Insgesamt waren sich die Diskutierenden einig, dass Bibliotheken agiler und experimentierfreudiger werden müssen.

- Citizen Science: Die breite Öffentlichkeit als wichtige Zielgruppe für Wissenschaftliche Bibliotheken?

Citizen Science wird nicht als Kernaufgabe von Bibliotheken angesehen, bietet aber dennoch Chancen. Denn Bibliotheken könnten eine Brücke zwischen der Öffentlichkeit und den Fachwissenschaftlerinnen und -wissenschaftlern schlagen und so Verständnis für das wissenschaftliche Arbeiten vermitteln.

- Wissenschaftliche Bibliotheken und Privatwirtschaft: Notwendig, sinnvoll oder verwerflich?

Einstimmige Meinung: Wenn die Rahmenbedingungen stimmen und Bibliotheken mit der Privatwirtschaft auf Augenhöhe zusammenarbeiten, ist nichts dagegen einzuwenden. Wichtig sind offene Systeme und Schnittstellen. Grundsätzlich werden insbesondere im IT-Bereich externe Kapazitäten benötigt, um Entwicklungen in Bibliotheken anzustoßen, wie das Beispiel Folio zeigt. ${ }^{5}$

\section{Funktioniert eine Tagung, die als Präsenzveranstaltung geplant war, auch online?}

Ein klares: Ja - mit einer kleinen Einschränkung, auf die wir am Ende eingehen möchten. Die Resonanz im Chat und in den sozialen Medien zur „Zukunft Wissenschaftlicher Bibliotheken?!“ fiel durchweg positiv aus - sowohl hinsichtlich der Inhalte als auch bezüglich des Formats. Auch ZB MED und Open Password zogen ein positives Fazit: Eine virtuelle Tagung bietet zahlreiche Vorteile, wie zum Beispiel die Beteiligung der Teilnehmenden durch Diskussionen im Chat oder die problemlose Dokumentation per Videoaufzeichnung. Zudem erlaubt das Format auch kleineren Bibliotheken mit weniger Zeit und geringerem Budget für Fortbildungen, an einer Tagung teilzunehmen. Das ist uns Motivation, weitere Online-Veranstaltungen zu planen.

Eine Kollegin hatte den Auftrag, sich nur um Twitter zu kümmern - auch auf diesem Kanal gab es unter dem

5 https://www.folio-bib.org/ [10.6.2020]. 


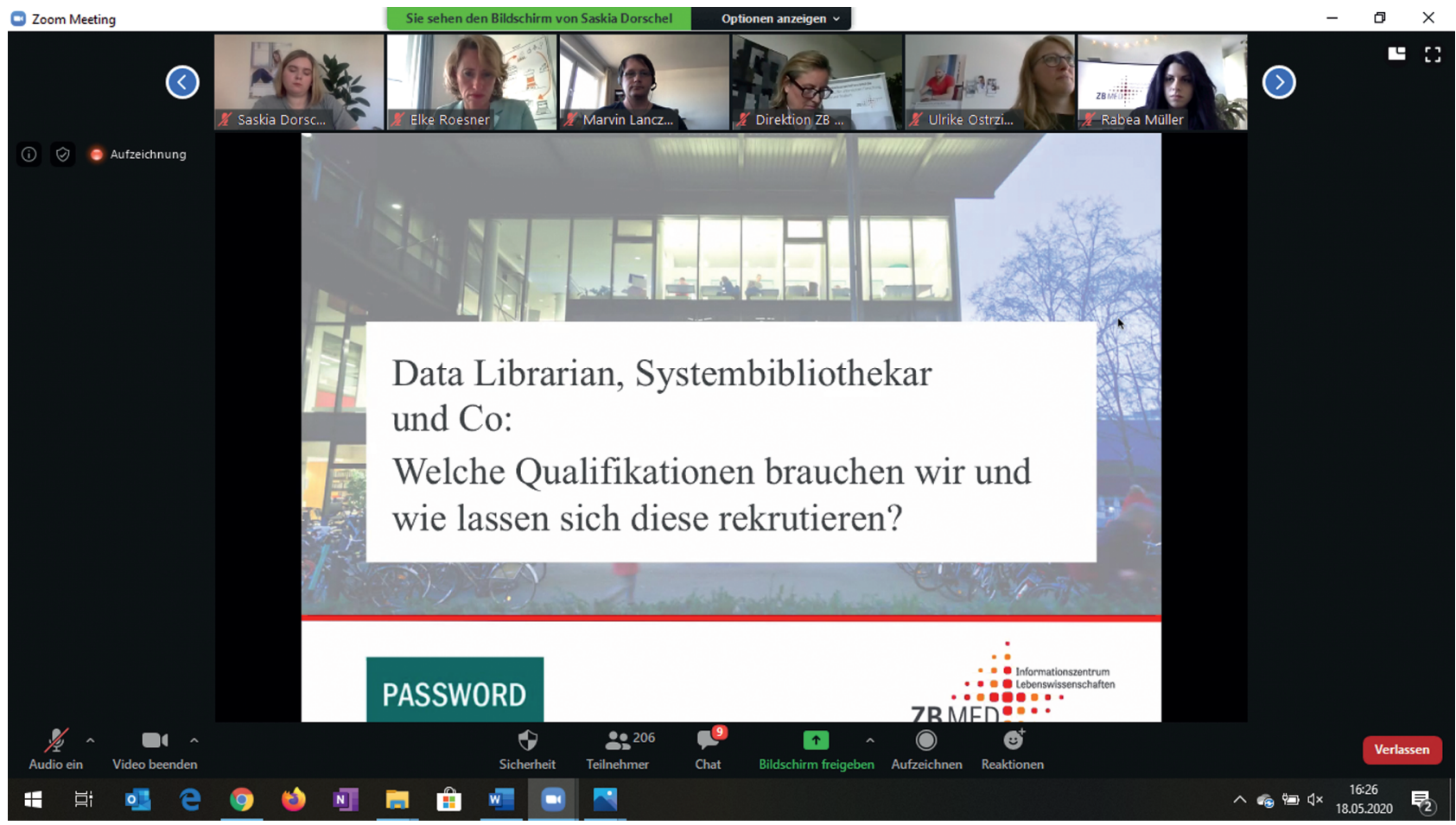

Abbildung 2: Screenshot mit dem Referenten Marvin Lanczek (ZBIW), Gabriele Herrmann-Krotz (Kaufmännisch-Administrative Leitung von ZB MED) sowie dem Organisationsteam von ZB MED: Saskia Dorschel, Elke Roesner, Ulrike Ostrzinski und Rabea Müller.

Hashtag \#ZukunftWB einen lebhaften Austausch. Die klare Verteilung der Zuständigkeiten erwies sich als goldrichtig, denn wir alle hatten - obwohl wir scheinbar nur dabei saßen - durch unsere jeweiligen Aufträge permanent zu tun. Zudem saßen wir an sechs verschiedenen Orten und konnten uns nur über unseren Chat im Messenger verständigen - aber das funktionierte perfekt.

Ein paar „Holperer“ gab es dennoch: So flog Miriam Albers, die wegen der Kinderbetreuung vom eher netzinstabilen Emsland aus moderierte, während der Veranstaltung dreimal aus dem Netz. Eine jeweils andere Person aus dem Orga-Team übernahm sofort die Rolle - durch den internen Austausch über den Messenger-Kanal gut darauf vorbereitet. Es mussten diverse Mikros an- und ausgeschaltet werden. Einmal wurde der Ton eines Referenten aus nicht erkennbaren Gründen plötzlich undeutlich. Bei der Schlussrunde tauschten wir uns im Hintergrund darüber aus, wer noch gefragt werden müsste, da die Moderatorin teilweise offline war.

Übrigens: Die Videos zur Veranstaltung stehen ab Juni auf YouTube bereit - wenn Sie den ZB MED-Kanal ${ }^{6}$ dort abonnieren, erhalten Sie eine Benachrichtigung über den Upload. Zudem können Sie die Vorträge zeitnah als Pod-

6 https://www.youtube.com/user/ZBMED [4.6.2020]. cast im Open Science Radio ${ }^{7}$ nachhören - wir informieren Sie auf dem ZB MED-Twitter-Kanal ${ }^{8}$, sobald die Beiträge online sind. Die Präsentationen und die Dokumentation des Chats finden Sie auf der ZB MED-Website9 .

\section{Gelernte Lektionen - unsere Best Practises für virtuelle Veranstaltungen}

- Besonders präzise Vorbereitungen (Checklisten, genaue Ablaufpläne)

- Möglichst stabile Technik und Menschen, die sie gelassen bedienen

- Genaues Briefing der Referentinnen und Referenten

- Gute und ausführliche Infos an die Teilnehmenden in der Einladung

- Ein funktionierendes Organisationsteam im Hintergrund, das spontan reagieren kann

$7 \mathrm{http://www.openscienceradio.org/} \mathrm{[4.6.2020].}$

8 https://twitter.com/ZB_MED [4.6.2020].

9 https://www.zbmed.de/ueber-uns/presse/neuigkeiten-aus-zb-me d/artikel/tagung-im-neuen-normal-zukunft-wissenschaftlicher-biblio theken-als-virtuelles-event-starke-vo/ [4.6.2020]. 
- Interessierte Zuhörerinnen und Zuhörer - denn das Interesse war deutlich zu spüren und zu lesen

- Chat und Twitter parallel zur Tagung

- Ein Messenger-Kanal für das Orga-Team für Absprachen im Hintergrund

- Verteilte Rollen nach besonderen Fähigkeiten mit klaren Aufträgen. Da so viel parallel auf verschiedenen Kanälen passiert, ist es unmöglich, mit ganzer Aufmerksamkeit alle Aktivitäten gleichzeitig aufmerksam $\mathrm{zu}$ verfolgen. Daher ist es unabdingbar, sich auf das Team verlassen zu können.

- Ein Vorabtermin quasi als Generalprobe mit den Vortragenden als Technik-Check und gleichzeitig als Warming-up für die Veranstaltung

- Gemischte Formate innerhalb der Konferenz: Vorträge und Statements, Diskussionen auf Basis des Chats

- Und fast am wichtigsten neben der Planung: ein positiver „Spirit“, Lust am Format sowohl bei den Vortragenden als auch beim Orga-Team.

Im Nachgang der Tagung haben wir uns als Orga-Team zum ersten Mal in dieser Konstellation und mit ausreichend physischem Abstand voneinander auf der ZB MEDTerrasse getroffen und unser persönliches Fazit gezogen: Wichtigster Erfolgsfaktor ist der genaue Plan und ein ausreichend großes und gut funktionierendes Team mit verteilten Rollen, wo alle wissen, dass auf die anderen Verlass ist, wenn etwas hakt. Wir waren ein tolles Team aus sechs Frauen und haben alle sehr fokussiert und engagiert daran gearbeitet, die Tagung so glatt wie möglich zu veranstalten.

Wir zumindest sind inzwischen sehr davon überzeugt, dass sich Konferenzen auch in den virtuellen Raum übertragen lassen und wollen es wieder tun. Ein Wermutstropfen wird dabei immer bleiben: Persönliche Gespräche und das Netzwerken kommen zu kurz, so dass virtuelle Tagungen Präsenzveranstaltungen nicht komplett ersetzen können. Unersetzlich ist das persönliche Miteinander und eine positive Grundstimmung, die nur durch analoge Begegnungen entsteht. Warum sonst sind schon viele gute Ideen bei Konferenzfeiern entstanden? Außerdem fehlt die physische Dimension, die unbewusst Informationen ins Gehirn transportiert und die Aufnahme und Wahrnehmung von Informationen fördert. Wenn wir die Wahl hätten, würden wir uns für die Zukunft eine 50/50-Mischung aus analog und virtuell wünschen.

Deskriptoren: Wissenschaftliche Bibliothek, Virtuelle Tagung, Tagungsbericht, Digitalisierung, Planung, ZB MED

\section{Saskia Dorschel}

ZB MED - Informationszentrum Lebenswissenschaften

Gleueler Straße 60

50931 Köln

marketing@zbmed.de

\section{Ulrike Ostrzinski}

ZB MED - Informationszentrum Lebenswissenschaften

Gleueler Straße 60

50931 Köln

marketing@zbmed.de

\section{Elke Roesner}

ZB MED - Informationszentrum Lebenswissenschaften

Gleueler Straße 60

50931 Köln

marketing@zbmed.de 